\title{
Gestión del mantenimiento mediante Six Sigma para la optimización de la productividad de la maquinaria y equipos diversos para una pyme
}

\section{Management of the maintenance through Six Sigma for the optimization of the productivity of the machinery and diverse equipment for an SME}

\author{
CHAVEZ-MEDINA, Juan†ं*, LUNA-FERNÁNDEZ, Víctor Genaro, SANTIESTEBAN-LÓPEZ, \\ Norma Angélica, VELÁZQUEZ-MANCILLA, Jorge Enrique
}

Universidad Politécnica de Puebla, Benemérita Universidad Autónoma de Puebla

ID $1^{\text {er }}$ Autor: Juan, Chávez-Medina / ORC ID: 0000-0002-1978-0620, CVU CONACYT ID: 417889

ID $1^{\text {er }}$ Coautor: Víctor Genaro, Luna-Fernández / ORC ID: 0000-0002-5438-6573, CVU CONACYT ID: 168205 |

ID $2^{\text {do }}$ Coautor: Noma Angélica, Santiesteban-López / ORC ID: 0000-0001-7700-4139, CVU CONACYT ID: 240825 |

ID $3^{\text {er }}$ Coautor: Jorge Enrique, Velázquez-Mancilla / ORC ID: 0000-0001-7835-6339, CVU CONACYT ID: 510054 |

DOI: $10.35429 / J I E .2019 .10 .3 .17 .27$

Recibido 01 de Noviembre, 2019, Aceptado, 13 de Diciembre, 2019

\begin{abstract}
Resumen
La aplicación de Six Sigma a la Gestión del Mantenimiento, para la optimización de la productividad de las maquinarias y equipos diversos para una pyme, utilizando la metodología del Six Sigma, qué está conformado por sus 5 fases DMAIC. Esta investigación desarrolla y aplica una estrategia combinada de TPM y Six Sigma en una PYME para erradicar un problema importante de CTQ. En este sentido, se destaca el enfoque empleado, las herramientas y técnicas utilizadas, evidenciando los ahorros que se lograron mediante la aplicación estructurada de un procedimiento combinado de TPM / DMAIC. A través de la aplicación correcta de los métodos TPM y Six Sigma, la presente investigación identifica los ajustes de parámetros óptimos y las actividades de mantenimiento, lo que permitió a la empresa erradicar los problemas de CTQ y lograr mejoras significativas en la calidad del producto, el costo y la entrega de un modesto desembolso financiero. La aplicación de la estrategia y las conclusiones resultantes en cuanto a su efectividad para la industria es el valor real de este trabajo, por lo cual, será valioso para profesionales de calidad, y especialistas en fabricación en una amplia gama de industrias
\end{abstract}

Calidad, Six Sigma, TPM, PYME

\begin{abstract}
The application of Six Sigma to Maintenance Management, to optimize the productivity of machinery and equipment for an SME, using the Six Sigma methodology, which is made up of its 5 DMAIC phases. This research develops and applies a combined strategy of TPM and Six Sigma in an SME to eradicate a major CTQ problem. In this sense, the approach used, the tools and techniques used are highlighted, evidencing the savings that were achieved through the structured application of a combined TPM / DMAIC procedure. Through the correct application of the TPM and Six Sigma methods, the present research identifies optimal parameter adjustments and maintenance activities, which allowed the company to eradicate CTQ problems and achieve significant improvements in product quality, the cost and delivery of a modest financial outlay. The application of the strategy and the resulting conclusions regarding its effectiveness for the industry is the real value of this work, for which, it will be valuable for quality professionals, and specialists in manufacturing in a wide range of industries.
\end{abstract}

Quality, Six Sigma, TPM, SMEs

Citación: CHAVEZ-MEDINA, Juan, LUNA-FERNÁNDEZ, Víctor Genaro, SANTIESTEBAN-LÓPEZ, Norma Angélica, VELÁZQUEZ-MANCILLA, Jorge Enrique. Gestión del mantenimiento mediante Six Sigma para la optimización de la productividad de la maquinaria y equipos diversos para una pyme. Revista de Ingeniería Industrial. 2019 3-10: 17-27

\footnotetext{
$\dagger$ Investigador contribuyendo como primer autor.
} 


\section{Introducción}

Six Sigma puede ser considerado tanto una estrategia empresarial y una ciencia para reducir costos de servicio, y crear mejoras significativas para la satisfacción del cliente y al mismo tiempo ahorro a través de combinar metodologías de un proceso estadístico y empresarial a un modelo integrado de proceso, producto y mejora de servicio. En Six Sigma las mejoras están definidas por su impacto encima de la satisfacción de cliente y del valor (Pande \& Holpp, 2002)

Los sectores económicos importantes para las inversiones son el crecimiento de las Pyme. Las condiciones para poder ser candidatos de aprobación para postularse en las licitaciones son grandes, por lo que la única manera que tienen las Pequeñas y Medianas empresas para poder desarrollarse dentro del sector y ser partícipes en las inversiones, son dos: asociándose con empresas grandes o ser subcontratistas de las empresas de otras empresas elegidas. En estas decisiones se incluyen a los equipos como adquisiciones, mantenimiento, reparaciones, reconstrucciones, eficiencias, disponibilidad, confiabilidad, reemplazos, costos y bajas de activos. Las empresas constructoras de nivel internacional están inmersas en lo que se denomina "El Mantenimiento de Clase Mundial", que significa tener políticas para realizar las labores cotidianas de trabajo con buenas prácticas tanto en el campo administrativo como en el campo técnico.

La competitividad de las empresas constructoras se le atribuye en adquirir la mejor rentabilidad por metro cúbico movido o por la máxima cantidad de horas máquina vendida. En el primer aspecto la máxima rentabilidad depende de la máxima producción horaria y del mínimo costo horario de máquina.

Por otro lado, el siguiente aspecto a considerar en la máxima rentabilidad dependerá de la mayor cantidad de horas trabajadas de la máquina y del menor costo horario de máquina.

Por lo anterior, una adecuada gestión del mantenimiento del equipo mecánico, es esencial para maximizar la rentabilidad empresarial, para ello es necesario contar con herramientas que ayuden en este proyecto.
Una situación real en este medio, muestra que el mantenimiento de equipos y máquinas en las pequeñas y medianas empresas de construcción consiste en minimizar los cambios de aceites a las máquinas y en algunas se realiza un continuo manejo administrativo de un programa de mantenimiento preventivo básico.

Para lograr la mejora en la eficiencia de los procesos a fin de mantener la disponibilidad y confiabilidad de los equipos, así como de los procesos del taller, será conseguida con el término empleado en control de calidad y es la mejora continua, el cual se considera el espíritu visionario de lograr la perfección. Para ello, utilizando el método administrativo Six Sigma, ayudará al análisis de los resultados de la gestión de mantenimiento y permitirán la mejora continua, así como el logro de los resultados esperados, traducidos en la eficiencia en el manejo del taller, mejora de la disponibilidad mecánica y aseguramiento de la vida útil de las máquinas.

Dentro de este contexto, es necesario indicar que, en México existen diversas empresas manufactureras que demandan calidad en sus productos y/o servicios; y en el ramo de materiales de la construcción no es la excepción, como las productoras de block, donde el mercado es cada vez más competitivo, debido a la proliferación de la oferta que se tiene de este producto, y principalmente en el estado de Puebla como el municipio de San Pedro Cholula y anexas, es por ello que la presente investigación plantea un modelo de gestión del mantenimiento mediante Six Sigma para la optimización de la productividad de las maquinarias y otras variables que afectan a la calidad en una PYME dedicada a la fabricación de materiales de construcción (bloquera).

\section{Fundamentos teóricos y definiciones de constructo}

Six Sigma fue introducida por primera vez en 1987 por la compañía Motorola dirigida por un equipo de directivos encabezados por Bob Galvin, presidente de dicha compañía con el propósito de reducir los defectos de productos electrónicos (Escalante et al. 2008).

Desde entonces, Six Sigma ha sido adoptada, enriquecida y generalizada por un gran número de compañías. 
En la literatura varios casos de aplicación exitosa como lo mencionan teóricos como Kaushik, et al. 2012; Timans, et al. 2012; Nicolay, et al. 2012; entre otros. Six Sigma está considerado como la evolución de las teorías clásicas de la calidad y la mejora continua, como Control Estadístico de Proceso (SPC) y la Administración de la Calidad Total (TQM) (Folaron, 2003; Maleyeff, et al. 2012). En este sentido, Six Sigma toma algunos elementos de sus teorías predecesoras y los organiza de forma sistemática, creando un enfoque mejorado y con mayor efectividad en la consecución de resultados.

En México, en los últimos años, son cada vez más las empresas Pymes que han aplicado la metodología Six Sigma, debido a que representa la mejora continua de sus procesos y por lo mismo un ahorro sustancial en sus finanzas (Domínguez, 2016).

Por otra parte, Six Sigma es una métrica que se emplea para indicar el número de DPMO (Defects per Million Opportunities, en inglés), o cómo se desempeña el proceso con respecto a las necesidades del cliente.

El cálculo de dicho número se realiza a través de las siguientes expresiones según Forrest W. Breyfogle en su obra "Implementing Six Sigma" (Bahena, 2006).

- $\quad$ Unidad (U): Es un artículo producido o procesado.

- Defecto (D): Cualquier evento que no cumpla la especificación de un CTQ (control total de calidad).

- Defectuoso: Una unidad que tiene uno o más defectos.

- Defectos por unidad (DPU): Es la cantidad de defectos en un producto

$\mathrm{DPU}=\mathrm{D} / \mathrm{U}$

- $\quad$ Oportunidad de defectos (O): Cualquier acontecimiento que pueda medirse y de una oportunidad de no satisfacer un requisito del cliente.

- $\quad$ Defectos por oportunidad (DPO):

$\mathrm{DPO}=\mathrm{D} /(\mathrm{U} \times \mathrm{O})$

- Defectos por millón de oportunidades (DPMO): Es el número de defectos encontrados en cada millón de unidades. Capacidad del proceso: Capacidad del proceso para cumplir especificaciones o requerimientos del cliente.
- Rendimiento estándar o de primera pasada (YFT): Es el porcentaje de producto sin defectos antes de realizar una revisión del trabajo efectuado y es la probabilidad de que una unidad pase el ensamble final con 0 defectos

$\mathrm{YFT}=(1-\mathrm{DPO}) * 100$

- $\quad$ Rendimiento al final o de última pasada (YLT): Es el porcentaje de producto sin defectos después de realizar la revisión del trabajo.

- Rendimiento Real o Estándar (YRT) mide la probabilidad de pasar por todos los subprocesos sin un defecto, se determina con el producto del resultado de cada paso: YFP1x YFP2x YFP3x ..... YFPn

- Rendimiento Normal (YN) mide el promedio de rendimientos por los pasos del proceso. Es el promedio exponencial basado en el número de pasos del proceso, no es un promedio aritmético. $\mathrm{YN}=\mathrm{n} \sqrt{ }$ YRT (Ec.4) (Hemant, 2011)

Donde $\mathrm{n}$ es igual al número de pasos en el proceso. Cabe señalar que la diferencia entre dpmu y dpmo es que una unidad puede tener varias oportunidades de cometer defectos. Por ejemplo, Si en cierto proceso se encontraron 10 defectos en una muestra de 100 unidades:

$-\quad \mathrm{dpu}=10 / 100=0.1($ defectos por unidad $)$

$-\quad \operatorname{dpmu}=($ dpu $)(106)=100,000($ defectos por cada millón de unidades)

$\mathrm{Si}$ en cada unidad existen 10 posibilidades de ocurrencia en un defecto.

$$
\begin{aligned}
& -\quad \mathrm{dpo}=10 / 1000=0.01 \\
& \text { - } \quad \mathrm{dpmo}=\mathrm{dpmu} / 10=10,000 \text { (defectos por } \\
& \text { cada millón de oportunidades) }
\end{aligned}
$$

Si cada unidad solamente tiene una oportunidad en la que puede ocurrir algún defecto, dpmo=dpmu. Originalmente ppm significa unidades defectuosas por cada millón, independiente mente del número de defectos en dichas unidades (Escalante, 2008).

El objetivo de la metodología Six Sigma es disminuir los defectos a la cantidad de 3.4 DPMO, y es muy importante que las empresas suban a estos niveles para poder ser competitivas a nivel mundial. 
La mayoría de las empresas operan a un nivel más bajo de calidad, por lo general entre 2 y 3 sigmas lo que significa entre 66,000 y 300,000 defectos por millón (Eckes, y Cárdenas, 2006).

\section{Optimización de procesos industriales}

La Optimización un proceso industrial significa mejorar el proceso y para ello se requiere utilizar o asegurar todos los recursos que intervienen en él de la manera más excelente posible. Dicha optimización está orientada hacia dos metas fundamentales: Maximizar ganancias y Minimizar costos, esto teniendo como consecuencia una mayor producción a un menor costo.

Por consiguiente el principal propósito de optimizar un proceso es incrementar la productividad. La optimización de procesos es una tarea difícil pero realizable, el cual se quiere de la colaboración y apoyo de todo el personal de la organización, trabajo en equipo.

El proceso de Optimizar implica en primer lugar poseer el conocimiento total del mismo, es decir, se necesita poseer toda la información relativa de las operaciones realizados en forma sistemática. (Boucly, 1999)

\section{Productividad}

La Productividad se define como la relación entre la cantidad de bienes y servicios producidos y la cantidad de recursos utilizados. En la industria la productividad sirve para evaluar el rendimiento de talleres, maquinaria, equipo de trabajo y los empleados.

La productividad de máquinas y equipos están dados como parte de sus características técnicas. En concreto, la productividad debe ser definida como el indicador de eficiencia que relaciona la cantidad de producto utilizado en la cantidad de producción obtenida. El mejor camino para que un negocio pueda crecer y aumentar su rentabilidad o sus utilidades es aumentando su productividad (Gutiérrez, 2007)

\section{Gestión de mantenimiento}

El objetivo del mantenimiento es el medio que tiene toda empresa para conservar la eficiencia y eficacia su activo fijo.
En global al conjunto de actividades necesarias para: Mantener una instalación o equipo en funcionamiento.

El mantenimiento incide en la cantidad de la calidad de la producción, por lo que propósito del mantenimiento es asegurar la disponibilidad planeada al menor costo dentro de las recomendaciones de garantía y uso de los fabricantes de los equipos e instalaciones y las normas de seguridad.

Se puede resumir que la gestión de mantenimiento tiene el propósito de aumentar la rentabilidad de las empresas, contribuyendo a que los equipos o sistemas estén disponibles para operar tanto tiempo como sea posible como también permitiendo en funcionamiento de los mismos a un costo unitario (Prieto, 2008).

Por lo anterior, es necesario mencionar que actualmente el Mantenimiento Productivo Total incorpora nuevos conceptos a la aplicación práctica del mantenimiento comparados con las generaciones anteriores, pues se incorpora a las actividades de mantenimiento a todos los operarios de producción y la participación activa de todos los empleados, además de agregar en su seno las prácticas del Mantenimiento Preventivo, Correctivo, Predictivo, las Mejoras y la Prevención del Mantenimiento, el TPM eleva el nivel de la eficacia del equipo mejorando los factores de disponibilidad, eficiencia del desempeño y el porcentaje de productos de calidad. El JIPM (Japan Institute of Plan Maintenance), define como el TPM como un sistema orientado a lograr cero accidentes, cero defectos y cero pérdidas (Depestre, 2012).

\section{Metodología Six Sigma}

La estrategia Six Sigma se basa en una metodología de cinco fases llamada DMAIC, es un acrónimo de los principales pasos dentro de la metodología, definir, medir, analizar, mejorar, y controlar. Se decidió que el proceso DMAIC formaría la base fundamental para la estrategia de TPM y por lo tanto el enfoque estándar para la adopción de las principales etapas del proyecto TPM.

Cada etapa se explica en detalle en la siguiente sección del documento. 


\section{Desarrollo}

Fase de definición

Se tuvo una plática con el director de la empresa donde se realizó el proyecto, en la que se dispuso que la problemática principal e interés de los directivos es la reducción del producto defectuoso en la fabricación de blocks prefabricados. Así como también tener una mejor distribución y colocación del producto en almacenamiento.

La información que se pudo obtener de la empresa fue la capacidad de la máquina, así como también, el porcentaje de producto defectuoso, la demanda y el costo del proceso de la fabricación de Block prefabricado. También se tuvo que observar el proceso y se obtuvieron datos más precisos.

El siguiente paso fue el definir qué tipo de limitaciones y qué tipo de alcances con el fin de llegar a un resultado más concreto. Uno de los alcances fue el proponer un nuevo procedimiento, así como también una nueva capacitación, y definir y concretar la línea de producción. En cuanto a las limitaciones sólo se pudo encontrar que el único impedimento era el presupuesto fijo para la inversión del proyecto. Además, que se desarrolló también para esta fase, la estructura de roles y responsabilidades, un diagrama SIPOC, un mapa de flujo de proceso detallado, un mapa gráfico de proceso y la descripción del proceso narrativo en forma secuencial.

Cabe señalar en este punto de limitaciones y alcances que no se podrán establecer controles totales, debido a que se lograra operativizar todas las variables, cuando se estabilice por completo el proceso en función a Six Sigma, porque lleva una curva de adaptación de que puede variar hasta un año (Escalante, 2008).

\section{Estructura organizacional, roles y responsabilidades}

En esta parte se describen las funciones que cada uno de los participantes. Para el desarrollo de Six Sigma en sus diferentes fases. Además, se establecen el tipo de actividades, y dónde estarán involucrados cada uno en cada fase.
Nos nombres de las personas que integraron el equipo de trabajo se omitieron intencionalmente, las cuales fueron, el Gerente General que es el responsable de aprobar el proyecto (Champion), Gerente de Producción. (Black Belt) y jefe de producción (Green Belt). Por lo cual, se le asignaron roles y responsabilidades en la que se describe claramente las labores y deberes que se deben cumplir.

\section{Principales productos que se elaboran en la empresa}

Existen en la empresa una gama de productos que se manufacturan, sin embargo, hay 2 tipos principalmente que abarcan el $48 \%$ de acuerdo con el historial de ventas de los ejercicios 20172018. Por otra parte, cabe señalar que el estudio también se enfoca en los productos mencionados a continuación, de acuerdo a la información proporcionada por la empresa

\section{Block Hueco}

Se utiliza para todo tipo de muros, su colocación es rápida, ahorro en cimbras por sus huecos se elaboran castillos integrados. Cada pieza pesa en promedio14 Kg. y tiene un rendimiento de 12.5 piezas por $\mathrm{m}^{2}$. Cuenta con una resistencia de 90 $\mathrm{Kg} / \mathrm{cm}^{2}$.

\section{Block Macizo}

Se utiliza para bardas, casas habitación, bodegas, naves industriales, etc. Cuenta con un mayor rendimiento al colocarlo por su tamaño. Cada pieza pesa en promedio $13 \mathrm{Kg}$. y tiene un rendimiento de 12.5 piezas por $\mathrm{m}^{2}$. Con una resistencia de $60 \mathrm{Kg} / \mathrm{cm}^{2}$.

Es importante mencionar también, que el proceso es habitualmente el mismo para la elaboración de los diferentes tipos de block; En la mayoría de los casos, sólo se cambia el molde del producto y el proceso prácticamente es el mismo.

\section{Descripción del Proceso}

Es importante conocer el proceso de producción para poder entender cómo se elaboran los blocks prefabricados de una manera rápida y comprobar los pasos de la producción.

Secuencia de producción de la fabricación de block mostrada en la figura 1

CHAVEZ-MEDINA, Juan, LUNA-FERNÁNDEZ, Víctor Genaro, SANTIESTEBAN-LÓPEZ, Norma Angélica, VELÁZQUEZMANCILLA, Jorge Enrique. Gestión del mantenimiento mediante Six Sigma para la optimización de la productividad de la maquinaria y 


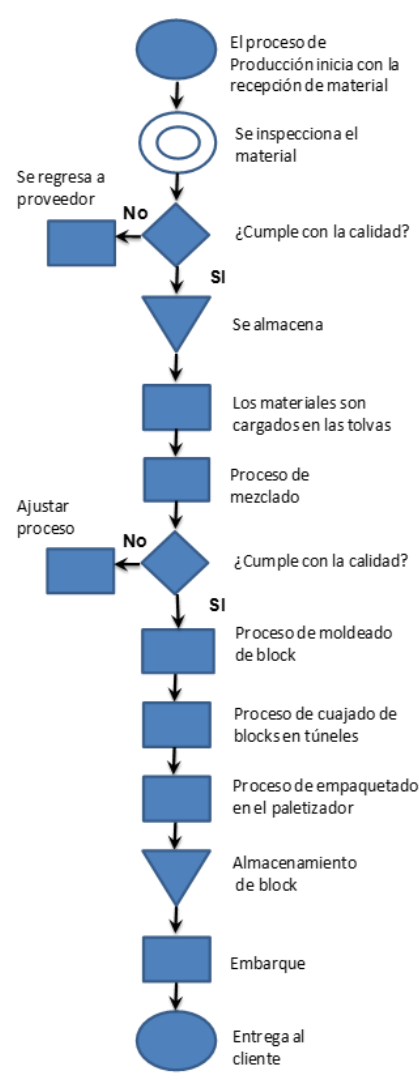

Figura 1 Diagrama de flujo de proceso Fuente: Elaboración propia

La etapa de definición desencadenó el desarrollo de un equipo TPM dentro de la empresa. Esta involucró la capacitación de los miembros del equipo en los principios de TPM, así como la implementación de un programa $5 \mathrm{~S}$ destinado a pilotar la limpieza autónoma y trabajo en equipo antes de emprender proyectos de mantenimiento específicos y específicos dentro del área de fabricación de block.

En esta fase de definición se ha descrito las particularidades básicas que se requieren en esta instancia, como los procesos que existen en las áreas productivas en la bloquera, donde se desarrolló la investigación, así como también se han identificó las personas que interactúan en el proceso directa o indirectamente, $\mathrm{y}$ se ha asignado los roles $\mathrm{y}$ responsabilidades del equipo de Six Sigma.

Así como también, se obtuvo información adicional a través de los diagramas antes mencionados que se elaboraron en esta fase, como los alcances y limitaciones del proceso, reproceso, tipos de clientes.

El paso siguiente de la metodología de Six Sigma es la fase de medición para diagnosticar la situación actual, identificar las necesidades e intervenir para poder reducir el número de productos defectuosos de blocks prefabricados.

\section{Fase de medición}

En esta etapa de medición se utilizaron técnicas para recolectar datos sobre el desempeño actual del proceso, haciendo el cálculo de los índices del proceso, se analizó la causa efecto del proceso a través del diagrama de Ishikawa (ver figura 2) determinando las posibles fuentes que provocan defectos como se muestra a continuación.

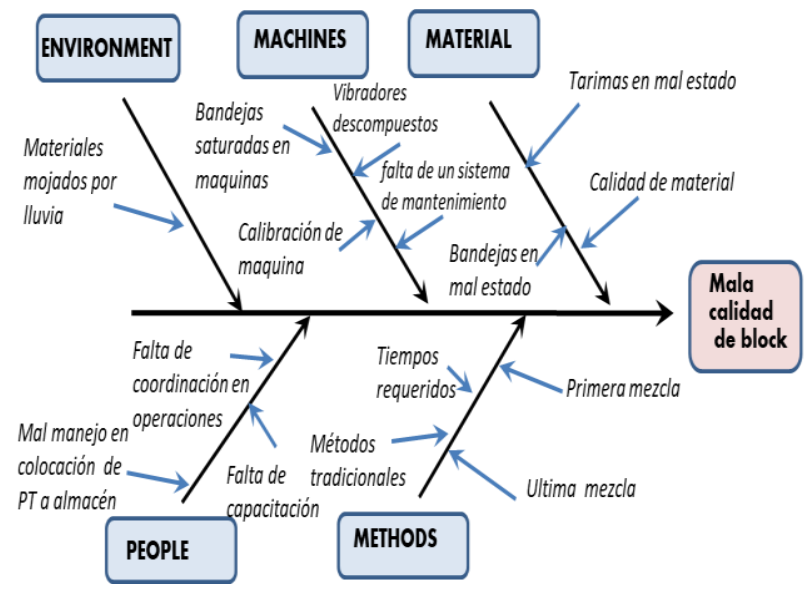

Figura 2 Diagrama de Ishikawa Fuente: Elaboración propia

En el diagrama de Ishikawa se muestran e Involucran elementos como mezcladoras, falta de capacitación, falta de coordinación de operaciones, bandejas saturadas y otras variables que causan la mala calidad en el block.

Por otra parte, se tienen datos del año 2017, proporcionados por la empresa. La producción anual de blocks prefabricados fue de $1,185,320$ unidades de las cuales, se tuvieron 19,325 piezas no satisfactorias clasificadas como defectos, dentro de las áreas de producción, así como también en la colocación del producto en el inventario.

Lo cual demuestra que existe un $1.63 \%$ de producto no deseado en el año 2009. Con esta información se calculó el DPMO (Defectos Por un Millón de Oportunidades), el cual fue de 16,303.61 y el nivel sigma del proceso, que se obtuvo fue de 3.64. Cálculo obtenido por Six Sigma calculator (Figura3). 


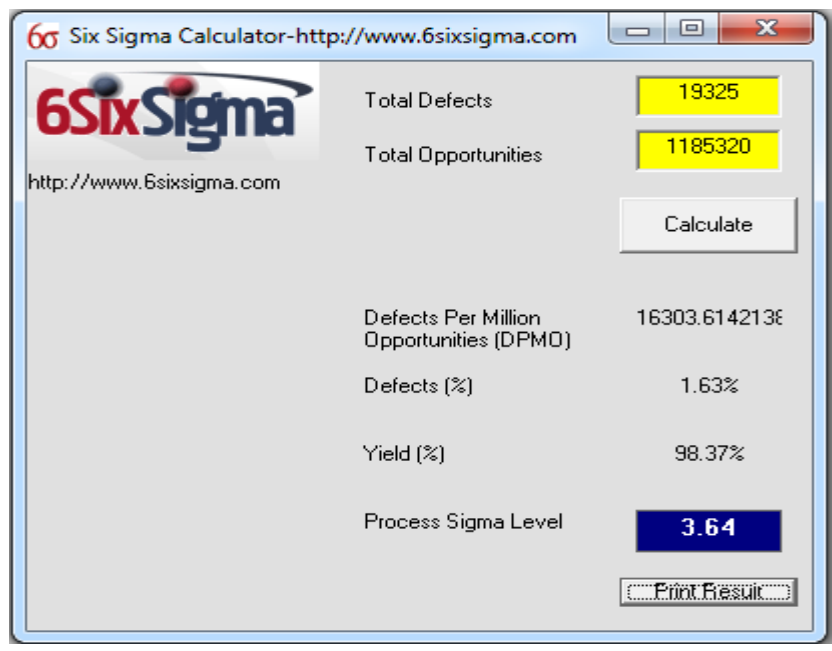

Figura 3 Calculadora Six Sigma, 6sixsigma.com

Los datos obtenidos en esta fase son parte fundamental, debido a que refleja la posición de la empresa dentro del contexto mundial en competitividad, de acuerdo a la Tabla 1.

\begin{tabular}{|c|c|c|c|c|}
\hline $\begin{array}{c}\text { Nivel } \\
\text { de } \\
\text { Sigma }\end{array}$ & $\begin{array}{l}\text { Defectos por } \\
\text { Millón de } \\
\text { Oportunidades }\end{array}$ & $\begin{array}{l}\text { Nivel de } \\
\text { Calidad }\end{array}$ & $\begin{array}{c}\text { Costo de } \\
\text { Calidad } \\
\text { Promedio }\end{array}$ & Clasificación \\
\hline 6 & 3.40 & $99.9999998 \%$ & $\begin{array}{l}\text { Menos del } \\
1 \% \text { de } \\
\text { Ventas }\end{array}$ & Clase Mundial \\
\hline 5 & 233.00 & $99.98 \%$ & $\begin{array}{l}5-10 \% \\
\text { de Ventas }\end{array}$ & $\begin{array}{l}\text { Industria } \\
\text { Promedio }\end{array}$ \\
\hline 4 & 6.210 & $99.4 \%$ & $\begin{array}{l}15-25 \% \\
\text { de } \\
\text { Ventas }\end{array}$ & $\begin{array}{l}\text { Baja } \\
\text { Competitividad }\end{array}$ \\
\hline 3 & 68.807 & $933 \%$ & $\begin{array}{l}25-40 \% \\
\text { de Ventas }\end{array}$ & $\begin{array}{l}\text { No } \\
\text { Competitivo }\end{array}$ \\
\hline 2 & 308.537 & $69.2 \%$ & No Aplica & $\begin{array}{l}\text { No } \\
\text { Competitivo }\end{array}$ \\
\hline 1 & 690.000 & $30.9 \%$ & No Aplica & $\begin{array}{l}\text { No } \\
\text { Competitivo }\end{array}$ \\
\hline
\end{tabular}

Tabla 1 Clasificación de las empresas por nivel de desempeño de Six Sigma

En la Tabla 1 se observa que el proceso se encuentra ubicado en 3.64 de DPMO en la escala de sigma, lo significa que no hay competitividad en la empresa, por lo tanto, es otro punto importante en tomar en cuenta para la implementación del modelo de calidad propuesto en el presente trabajo de investigación.

Por otra parte, también se encontró información adicional con la aplicación del diagrama de Ishikawa (causa y efecto), cuando se aplicó en las áreas que no se consideraban de suma importancia pero que afectan al producto final como la fórmula de los agregados, el paletizador, la primera mezcla y la lluvia que afecta a los agregados, entre otros.
Es importante también mencionar que los problemas que afectan en mayor grado a la mala calidad de block se ocasionan principalmente por las bandejas que se encontraban en mal estado, la última mezcla de la producción diaria, los problemas de la máquina, y la colocación de los blocks en el almacén. Para ello, se revisó el historial del 2017, donde se desglosaron los defectos más importantes encontrándose que las bandejas que están en mal estado ocasionaban un desperdicio que en conjunto con los defectos originados por problemas en maquinaria originan $49.62 \%$ de desperdicios totales. Un mal manejo de block en el almacén y falta de capacitación de operarios ocasionan un $37 \%$ de total de desperdicios como principales problemas (ver figura 6).

\section{Medición del proceso}

Para determinar la problemática del proceso en condiciones actuales, y verificar la funcionalidad del mismo, se recurrió a la recolección de datos para realizar posteriormente, un análisis estadístico con los datos obtenidos de un mes de producción.

El análisis de la información recabada que se realizó en conjunto con el equipo de Six Sigma, descrito a continuación en la fase de análisis, siguiendo el procedimiento metodológico de Six Sigma.

\section{Fase de análisis}

Una vez empleadas las herramientas de calidad como el diagrama de Pareto y la técnica de los "5 Por qué", se obtuvieron los elementos requeridos, de la fase de análisis de acuerdo con la metodología Six Sigma (Sirvent, et al. 2006), y con ello se continuó con la siguiente fase de mejora.

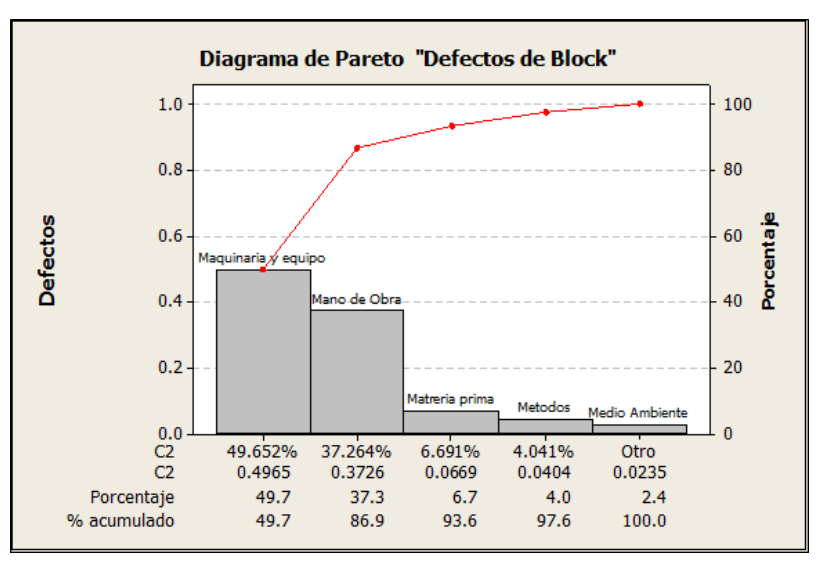

Figura 4 Pareto de defectos 
En relación a los datos de la figura 4 se analizó las causas de fallas en maquinaria con un historial de enero a sepriembre de 2017 como lo muestra la figura 4 y 5 .

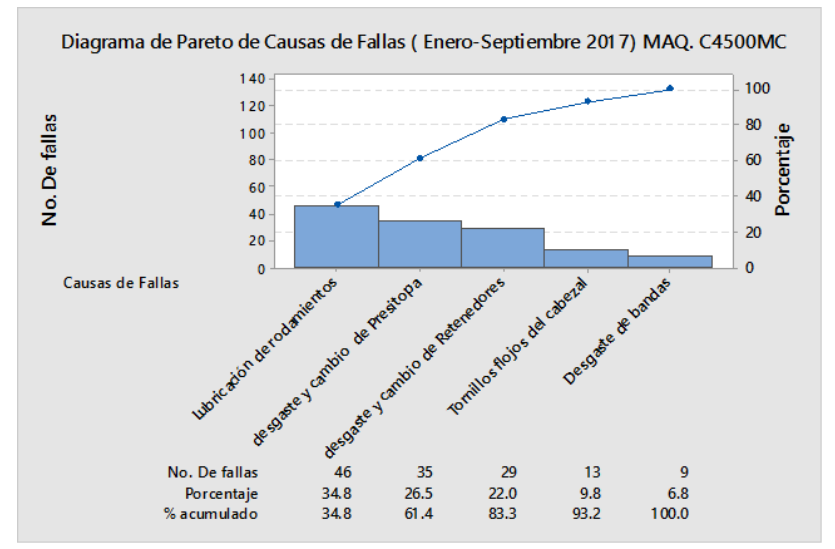

Figura 5 Pareto de defectos fallas de maquinaria

\section{Fase de Mejora}

Continuando con las fases de definición, medición y análisis, se procedió a emplear la etapa de mejora, aplicando la herramienta de calidad Brainstorming o lluvia de ideas, realizada en mayo de 2017, respetando la metodología de la herramienta, en este sentido, se escogió entre otras aplicables a esta fase, debido a que se tenían que desarrollar las posibles soluciones, que demanda esta etapa, por ello también el Brainstorming se enfocó principalmente en los defectos originados por mano de obra, equipo y maquinaria; con la participación del equipo de trabajo de Six Sigma. En función a la representación del mayor porcentaje de defectos, con base a la información del diagrama de pareto que se empleó en la fase anterior.

En este sentido se empleó un programa de capacitación para el personal operativo involucrado en el proceso en función a las necesidades de mejora de la mano de obra; y para la maquinaria y equipo se realizó un ajuste a la máquina de bolck y un programa de mantenimiento preventivo para los cuales se realizaron diferentes controles.

Por otra parte, también, se rediseño un método de trabajo necesario para la mejora y disminución de los defectos y desperdicio de material, así como también se realizaron controles para la recepción de la materia prima, y producto terminado.
Aunado a lo anterior se recurrió al mantenimiento productivo total, para maximizar la disponibilidad del equipo y maquinaria productiva de manufactura, impidiendo las fallas imprevistas y los defectos originados por esta causa; a través de la participación de los operadores en producción los cuales efectúan el mantenimiento autónomo a sus equipos y maquinas tales como lubricación, limpieza, y pequeños arreglos, así como el reporte de "sonidos raros" y con ayuda del análisis del historial de las máquinas y con la retroalimentación de los equipos de operación y mejora Kaizen.

De la misma forma, también se trabajó con los proveedores principalmente en los aspectos como especificaciones de material, condiciones de entrega, y necesidades de abastecimiento, para hacer el proceso cliente-proveedor más eficaz; además de lo mencionado se realizó una comparación antes y después de implementar el modelo Six Sigma que se enunciara propiamente en el siguiente capítulo de resultados de las mejoras del proceso en los defectos y desperdicios del proceso del block.

De igual manera comenzó con algo básico pero esencial dentro del rubro de mantenimiento en las máquinas de acuerdo a la tabla 2.

\begin{tabular}{|l|c|l|}
\hline \multicolumn{1}{|c|}{ Defectos de } & $61.90 \%$ & $\begin{array}{l}\text { Subricación mensual } \\
\text { de rodamientos y } \\
\text { chequeo de presitopa } \\
\text { mensual }\end{array}$ \\
\hline $\begin{array}{l}\text { Lubricación dedamientos- desgaste } \\
\text { y cambio de presitopa }\end{array}$ & $31.43 \%$ & $\begin{array}{l}\text { Chequeo y cambio de } \\
\text { retenedores, } \\
\text { verificando cada fin } \\
\text { de semana el ajuste de } \\
\text { turcas por vibración. }\end{array}$ \\
\hline $\begin{array}{l}\text { Desgaste } \\
\text { retenedores-tornillos } \\
\text { flojos del cabezal }\end{array}$ & $93.33 \%$ & \\
\hline$\%$ de mayores fallas & & \\
\hline
\end{tabular}

Tabla 2 Fallas mayores y su solución

\section{Fase de Control}

Esta fase consiste en diseñar y documentar controles necesarios para asegurar que lo conseguido de acuerdo a los cambios realizados.

\section{Implementación de controles}

Es importante señalar que en la elaboración de controles se tiene la siguiente limitante, de que no se ha completado en su totalidad la implantación de Six Sigma de acuerdo a lo explicado anteriormente en alcances $y$ limitaciones. 
Debido a que se tienen algunas restricciones de aprobación para realizar ciertos cambios que se explicaran en el siguiente capítulo de resultados y discusión. Sin embargo, hasta esta parte del trabajo se plantearon controles en la entrada de materia prima, en el proceso manejando control estadístico de proceso y seguimiento a través de gráficos, control de mantenimiento a través de mantenimiento preventivo a las máquinas y diario de TPM del operador (ver tabla 3), capacitación periódica del personal, Así como exámenes al personal operativo y formación de círculos de calidad.

\begin{tabular}{|c|}
\hline $\begin{array}{l}\text { Mantenimiento preventivo } \\
\text { Diario TPM del operador } \\
\text { MP Del Operador }\end{array}$ \\
\hline 1. Chequear si tuercas y pernos están bien ajustados \\
\hline 2. Chequear si los rodamientos han sido lubricados. \\
\hline 3. Chequear si las bandas están en perfecto estado. \\
\hline $\begin{array}{l}\text { 4. Chequear si escotilla de vaciado y tolva de llenado } \\
\text { están en buen estado. }\end{array}$ \\
\hline $\begin{array}{l}\text { 5. Chequear si el motor eléctrico está limpio, en buen } \\
\text { estado y los cables que estén protegidos. }\end{array}$ \\
\hline $\begin{array}{l}\text { 6. Chequear si el medidor de dureza y calibración de } \\
\text { compactación es correcto. }\end{array}$ \\
\hline $\begin{array}{l}\text { 7. Chequear si el engranaje principal funciona } \\
\text { correctamente }\end{array}$ \\
\hline
\end{tabular}

Tabla 3 Diario TPM del operador Fuente: Elaboración propia

\section{Discusión de resultados}

Después de haber trabajado para instigar un proceso para identificar las oportunidades para mejorar la calidad y reducir la variabilidad utilizando herramientas estadísticas, se obtuvieron 1164 defectos de una producción de 192,570 blocks, lo que matemáticamente se determinan 6044. 55 DPMO.

En la fase de medición se acordaron las variables que se necesitaban medir y la forma con que se calculó fue a través de los diagramas de Ishikawa, Pareto, el valor de Six Sigma. El cálculo de los DPMO al inicio del estudio fue de 15,638.11 los cuales fueron determinados matemáticamente de acuerdo a la hoja de recolección de datos del mes de noviembre del 2017.

En la fase de mejora se calcularon los ahorros obtenidos en el proyecto de aproximadamente 6044 defectos por millón de oportunidades, así como ahorro en la mano de obra equivalentes a $82.88 \%$.
El cálculo del ahorro aproximado que la empresa tendrá en acorto plazo en cuanto a defectos en otros rubros es de $67 \%$ en materia prima, $25 \%$ de maquinaria y equipo, métodos de trabajo $78 \%$, y medio ambiente $35 \%$.

Por lo que respecta a la fase de control se estableció para el personal operativo un programa de capacitación la cual se midió por medio de un examen; en cuanto a la maquinaria y equipo se manejó un programa de mantenimiento productivo total donde se implementaron los controles diferentes para evitar las fallas que originaban defectos.

Esta metodología resultó exitosa ya que administrativamente los cambios por aceptación se vieron reflejados en los datos de la fase de análisis y a la utilización de métodos de análisis estadístico y matemático.

El modelo también permite a la empresa para ayudar a identificar la necesidad de contar con un equipo multidisciplinario que trabaja estratégicamente, (Pyzdek, 2014; De Mast, 2004; Thomas y Antony, 2004).

\section{Conclusiones y recomendaciones}

Utilizando Six Sigma de manera adecuada, DMAIC permitió hacer frente a problemas de mantenimiento y defectos de calidad. Del mismo modo, los resultados también pueden proporcionar el estímulo para la aplicación más amplia de la técnica para crear mejoras en los procesos minimizando costos.

Para algunas organizaciones conciben a Six Sigma como un mito exclusivo para grandes empresas, sin embargo, se empieza a emplear este modelo de calidad en la PYMES para reducir desperdicios, y en el caso particular de este trabajo resultó buena la implantación para la reducción de desperdicios en la productora de blocks, siendo una empresa pequeña con tendencia de crecimiento.

A continuación, se enumeran las conclusiones y recomendaciones del presente trabajo de forma ordenada y sistemática.

1. El desarrollo del enfoque Six Sigma amplió una cultura hacia la mejora continua y la aplicación sistemática del sistema en toda la organización. 
2. La herramienta Six Sigma permitió a la empresa desarrollar métodos de los sistemas de cartografía y análisis avanzados y llegar a ser generalmente más "técnica" en su acercamiento a la resolución de problemas.

3. La implementación exitosa del marco integrado propuesto de Six Sigma proporciona un impulso para traer un cambio cultural en la empresa con la aplicación sistemática del enfoque integrado en toda la organización.

4. Six Sigma se llevó a cabo con el fin de mejorar las medidas de calidad, costo y entrega de la empresa. En todas las medidas, logró mejoras significativas, de 3,64 a 5,6 sigmas en 2018, y está constantemente avanzando en la escala.

En este sentido, se debe mencionar también que el ambiente de trabajo ha mejorado debido que se adoptó una cultura 5'S y con ello ajusto al cambio requerido, debido a que se comprendió la necesidad de generar esas mejoras en la empresa y el involucramiento de todo el personal ayudó a generar un flujo constante de ideas que contribuyeron a la mejora continua, logrando una mayor competitividad en el mercado, lo cual ha generado un crecimiento económico en la organización.

Finalmente es necesario mencionar que debido a la escasa evidencia en la literatura sobre casos de éxito en MYPES, y específicamente de empresas con giro de materiales para construcción como bloqueras, se recomienda que la investigación adicional se contraste con la investigación actual, lo cual, ayudará a tener una perspectiva holística.

\section{Referencias}

Bahena Q. M. (2006). Aplicación de la metodología seis sigma para mejorar la calidad y productividad de una planta de bebidas. Tesis de Maestría. México, Puebla: Universidad Iberoamericana.

Boucly, F. (1999). Gestión de Mantenimiento. Madrid: AENOR.

De-Mast, J. (2004), “A methodological comparison of three strategies for quality improvement", International Journal of Quality \& Reliability Management, Vol. 21 No. 2, pp. 198-213.
Depestre, L. O. L., \& Del-Centro, E. G. (2012). Del Mantenimiento Correctivo al Mantenimiento Centrado en la Confiabilidad. Centro Azúcar, 39(3), 7-14.

Domínguez, R. I. G., Amaya, P. P., López, R. R., Arredondo, R. D. M., Valdiviezo, I. C., \& Sáenz, A. G. (2016). Reducción de costos en pequeñas y medianas empresas con un enfoque Seis Sigma: Revisión de Literatura. CULCyT, (57).

Eckes, G., Cárdenas, N. J. (2006). El Six Sigma para todos. Colombia: Editorial Norma.

Escalante, E. J. (2008). Seis - Sigma. Metodología y Técnicas (1ra Ed.). México: Editorial Limusa.

Folaron. J. (2003). "The Evolution of Six Sigma". Six Sigma Forum Magazine. Vol. 2, Issue 4.

Gutiérrez, H. (2007). Control estadistico de calidad y Seis Sigma. México, D.F.: McGrawHillEducacion.

Hemant, U. (2011). Six Sigma para la excelencia empresarial: enfoque, herramientas y aplicaciones. Pearson Education India.

Kaushik, P., Khanduja, D., Mittal, K., \& Jaglan, P. (2012). A case study: Application of Six Sigma methodology in a small and mediumsized manufacturing enterprise. The TQM Journal, 24(1), 4-16.

Maleyeff, J., Arnheiter E., Venkateswaran, V. (2012). "The continuing evolution of lean Six Sigma". The TQM Journal. Vol. 24, Issue 6, DOI: $10.1108 / 17542731211270106$.

Nicolay, C. R., Purkayastha, S., Greenhalgh, A., Benn, J., Chaturvedi, S., Phillips, N., \& Darzi, A. (2012). Systematic review of the application of quality improvement methodologies from the manufacturing industry to surgical healthcare. British Journal of Surgery, 99(3), 324-335.

Pande, P. S., \& Holpp, L. (2002). ¿Qué es seis sigma? McGraw Hill.

Pyzdek, T., \& Keller, P. A. (2014). The Six Sigma handbook (Vol. 4). New York: McGrawHill Education. 
Sirvent, P., Mateu, J., Sagasta, P. S. (2006). Manual de control estadístico de calidad (3ra Ed.). España: Publicacions de la Univesitat Jaume I.

Thomas, A.J. and Antony, J. (2004), “Applying Shainin's variable search methodology inaerospace applications", Journal of Assembly Automation, Vol. 24 No. 2, pp. 184-91.

Timans, W., Antony, J., Ahaus, K., \& Solingen, R. (2012). Implementation of Lean Six Sigma in small-and medium-sized manufacturing enterprises in the Netherlands. Journal of the Operational Research Society, 63(3), 339-353. 